\title{
"Superscan" in diffusion-weighted imaging with background body suppression magnetic resonance imaging
}

\author{
Fumio Shimada MD PhD, Miwa Misawa MD, Tomio Suzuki MD PhD
}

— Cite as: CMAJ 2021 January 11;192:E48. doi: 10.1503/cmaj.191188

A 78-year-old woman attended the emergency department of a hospital with right femoral pain that had persisted for 2 weeks. Magnetic resonance imaging (MRI) showed suspected bone tumours, and the patient was referred to our hospital. Her pain was aggravated whenever she stood or walked and had spread throughout her body 4 weeks after onset. Because metastatic bone tumours were suspected, we performed whole-body diffusion-weighted imaging with background body suppression (DWIBS) MRI, which showed a diffused, high-signal intensity in multiple bones, resembling "superscan." Superscan is a phenomenon first described in relation to findings of diffuse symmetrical increased uptake of radionucleotides in bone but not kidney and bladder seen on technetium-99m diphosphonate bone scanning, which could point toward metastatic bone disease, metabolic bone disease or widespread primary bone disease ${ }^{1}$ (Figure 1A).

During the patient's hospital stay, her serum alkaline phosphatase level (311 IU/L [reference range 106-322 IU/L]) was normal and calcium level (10.8 mg/dL [reference range 8.4-10.1 mg/dL]) was almost within the normal range. Therefore, we considered diffuse osteolytic disease unlikely and attributed the MRI findings to bone marrow disease from metastasis caused by an unknown malignant growth. We performed 18-fluorodeoxyglucose positron emission tomographycomputed tomography (PET/CT) to search for the primary lesion, and the resulting images, acquired 13 days after MRI, showed diffuse uptake in the bone, similar to the DWIBS findings (Figure 1B). After a bone biopsy, we diagnosed primary bone marrow B-cell lymphoma.

The DWIBS imaging method was developed to detect tumours throughout the body using diffusion-weighted MRI. ${ }^{2}$ It has a reported detection rate equal to or better than that of bone scintigraphy for bone metastasis ${ }^{3}$ and is completed in half the time required by PET/ CT and at one-sixth of the cost (in Japan). It is particularly indicated in patients who wish to avoid radiation, are breastfeeding or pregnant.

\section{References}

1. Osmond JD III, Pendergrass HP, Potsaid MS. Accuracy of 99mTC-diphosphonate bone scans and roentgenograms in the detection of prostate, breast, and lung carcinoma metastases. Am J Roentgenol Radium Ther Nucl Med 1975;125:972-7.

2. Takahara T, Imai Y, Yamashita T, et al. Diffusion weighted whole body imaging with background body signal suppression (DWIBS): technical improvement using free breathing, STIR and high-resolution 3D display. Radiat Med 2004;22:275-82.

3. Stecco A, Lombardi M, Leva L, et al. Diagnostic accuracy and agreement between whole-body diffusion MRI and bone scintigraphy in detecting bone metastases. Radiol Med (Torino) 2013;118:465-75.
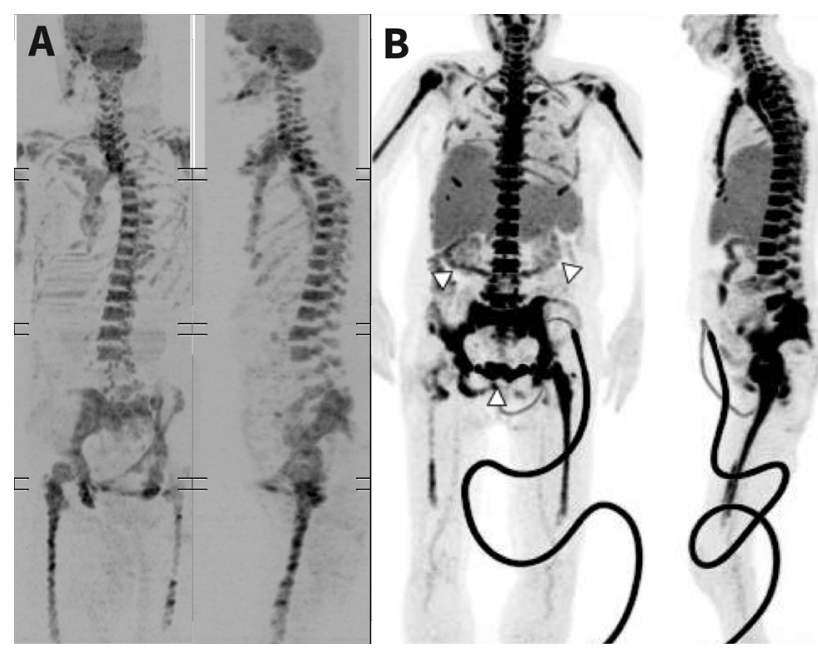

Figure 1: (A) Diffusion-weighted images with background body suppression (DWIBS; inverted black-and-white greyscale) in a 78-year-old woman with primary bone marrow B-cell lympoma, showing high signal intensities (in black) in the mandible, vertebral body, clavicle, sternum, humeral head, pelvis, femur, and ribs. (B) 18-Fluorodeoxyglucose positron emission tomography-computed tomography showing radionuclide uptake in the patient's mandible, vertebral body, clavicle, sternum, humerus, part of the ribs, pelvis and femur. Hepatosplenomegaly can also be observed and decreased radionuclide uptake in the kidneys and bladder (white arrowheads). The artificial arc on the lower body is an indwelling urinary catheter.

\section{Competing interests: None declared.}

This article has been peer reviewed.

The authors have obtained patient consent.

Affiliation: Department of General Medicine, Osaka Medical College Hospital, Takatsuki, Osaka, Japan

Funding: This study was funded by the Department of General Medicine, Osaka Medical College Hospital.

Content licence: This is an Open Access article distributed in accordance with the terms of the Creative Commons Attribution (CC BYNC-ND 4.0) licence, which permits use, distribution and reproduction in any medium, provided that the original publication is properly cited, the use is noncommercial (i.e., research or educational use), and no modifications or adaptations are made. See: https://creativecommons.org/licenses/by-nc-nd/4.0/

Correspondence to: Fumio Shimada, gmd012@osaka-med.ac.jp 